\title{
Leveraging Differential Chromatic Refraction in LSST
}

\author{
Gordon Richards (Drexel), Christina Peters (Toronto), \\ Bee Martin (Drexel), J. Bryce Kalmbach(UW), John Parejko (UW), \\ Ian Sullivan (UW), Franz E. Bauer (PUC/SSI)
}

November 30, 2018

\begin{abstract}
Our goal is to increase the accuracy of photometric reshifts (and object classification) in LSST by suggesting cadence changes that will increase the amplitude of the effect of differential chromatic refraction (DCR). This improvement could be realized by either extending the observing season or through a dedicated twilight campaign. We provide a metric for determining the expected photo- $z$ improvement for quasars for different opSims.
\end{abstract}

\section{White Paper Information}

- Contact author: Gordon Richards (Drexel University), gtr@physics.drexel.edu

\section{- Science Category:}

This white paper addresses issues related to classification and photometric redshifts of objects with intrinsic spectral breaks and strong emission features (e.g., AGN, galaxies, $\mathrm{SNe}$ ). Thus it is related to any science programs that depend on the discovery of such sources and is most directly related to the themes of "Exploring the Changing Sky" and "The Nature of Dark Matter and Understanding Dark Energy".

- Survey Type Category: Primarily related to extending the benefit of the WFD survey area, but is informed by (and could potentially benefit) the DDF surveys as well.

- Observing Strategy Category: Other. The proposed strategy is not a suggestion for a specific cadence or set of pointings (or the combination of the two), but rather is about optimizing the results given choices driven by other considerations. 


\section{Scientific Motivation}

LSST's lack of a spectroscopic component makes determination of accurate photometric redshifts all that much more important. In this white paper we point out the need not for better templates or algorithms, but rather for using all of the information that is available to help the photo- $z$ effort. In particular, we advocate for active consideration of the potential power of astrometric calibration in the photo- $z$ endeavor - in the form of including "differential chromatic refraction" (DCR; Kaczmarczik et al. 2009; Peters et al. 2015; Lee et al. 2018; Sullivan 2018).

DCR causes the position of an object to shift on the sky by different amounts in different bandpasses (according to the effective wavelength of the SED convolved with the filter response). Spectral features cause an anomalous shift (which is redshift dependent). This effect is illustrated for SDSS quasars in Figure 1 (Figure 4 from Kaczmarczik et al. 2009). In essence, DCR turns the Earth's atmosphere into an R 4 prism, where the resolution depends on the airmass of the observations. We can take advantage of the resulting DCR information to improve photo- $z$ estimates for objects with spectral breaks or strong emission lines in the $u$ or $g$ bands.

As described in Sullivan (2018), observing a field at a range of airmasses makes it possible to divide the $u$ or $g$ bands (possibly even $r$ ) into multiple sub-bands and estimate the flux of every pixel in each sub-band. We can then create a sort of low-resolution spectrum of every source within a single band, which aids in stellar classification and identification of quasars. These sub-band images will also be used to generate DCR-matched templates for image differencing for the Alert Generation pipeline for the main wide, fast, deep (WFD) survey of LSST. Including a few high airmass observations will improve the fidelity of the sub-band images, and improve the quality of the template images.

Figure 9 from Kaczmarczik et al. (2009) is reproduced in Figure 2, it illustrates that astrometric DCR adds spectral information otherwise hidden in the photometric colors. Thus, having more observations at high airmass can improve photo- $z$ (see Peters et al. 2015, Figure 14). LSST can achieve this goal in one of two ways: 1) by adding high airmass observations (e.g., in twilight), or 2) extending the observing season (which naturally produces more high airmass observations).

The purpose of this proposal is to illustrate the gains in photo- $z$ accuracy that can be achieved for AGNs - as an example of the overall gains that might be achieved for sources with spectral breaks or emission-line features in the $u$ or $g$ bandpasses. However, DCR will also yield improvements in object classification. As the classification metric is harder to quantify without full simulations, we concentrate here on illustrating the gains that can be achieved for photo- $z$. 


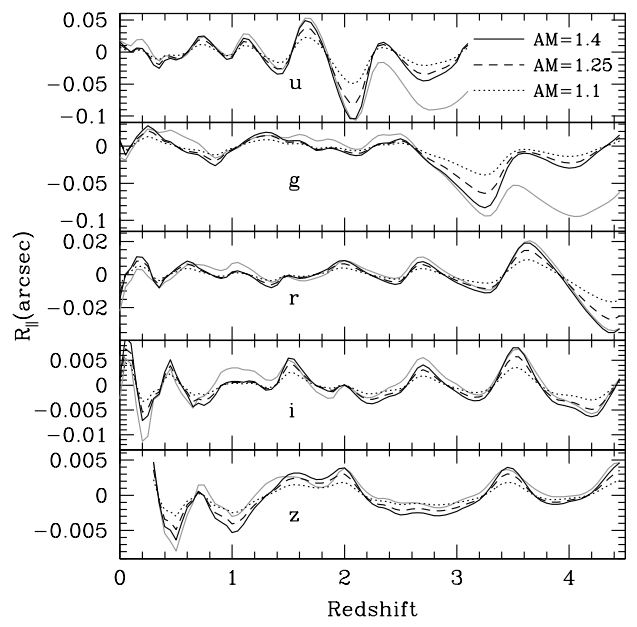

Figure 1: Figure 4 from Kaczmarczik et al. 2009. Expected change of quasar position on the sky as a function of redshift and bandpass. Each panel shows one of the ugriz filters and its expected positional change. Three airmass values are shown in black $(\mathrm{AM}=1.4$ : solid; $\mathrm{AM}=1.25$ : dashed; $\mathrm{AM}=1.1$ : dotted).

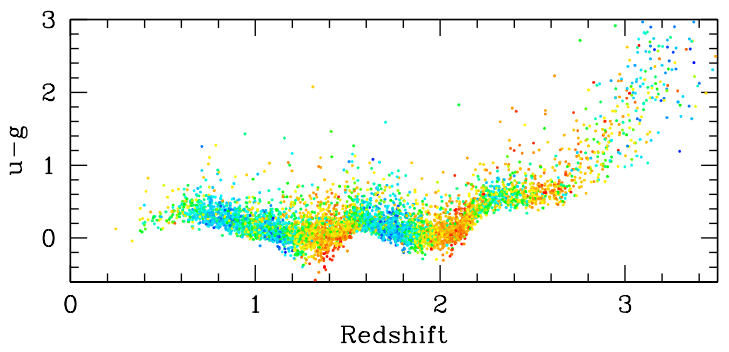

Figure 2: Figure 9 from Kaczmarczik et al. (2009). $u-g$ color as a function of redshift for SDSS quasars, color-coded by their DCR positional offsets (cool colors having a positive offset and warm colors having a negative offset). While an emission line moving through a bandpass produces a color change that is roughly symmetric with redshift, the positional offsets break this symmetry, allowing potentially significant improvements to photometric redshifts. 


\section{Technical Description}

Describe your survey strategy modifications or proposed observations. Please comment on each observing constraint below, including the technical motivation behind any constraints. Where relevant, indicate if the constraint applies to all requested observations or a specific subset. Please note which constraints are not relevant or important for your science goals.

\subsection{High-level description}

Describe or illustrate your ideal sequence of observations.

We advocate for the longest possible observing season in the WFD area-in order to increase the number of high airmass observations (AM > 1.5). Alternatively, LSST could undertake a high-airmass $g$-band twilight survey to increase the dynamic range in airmass of all of the survey fields.

Thus this white paper is both a specific proposal for such a twilight survey and also a general suggestion for choosing the optimal LSST cadence in the WFD. More high-airmass observations will improve the DCR signal and enable more robust object classification and photometric redshift estimates.

\subsection{Footprint - pointings, regions and/or constraints}

Describe the specific pointings or general region (RA/Dec, Galactic longitude/latitude or Ecliptic longitude/latitude) for the observations. Please describe any additional requirements, especially if there are no specific constraints on the pointings (e.g. stellar density, galactic dust extinction).

We are advocating for each region of the WFD to be observed one or more times in the $g$-band at "high" airmass during twilight. These observations can be spread over the full lifetime of the survey, but the earlier they occur, the sooner their information can be folded in to the algorithms.

\subsection{Image quality}

Constraints on the image quality (seeing).

Nominal WFD.

\subsection{Individual image depth and/or sky brightness}

Constraints on the sky brightness in each image and/or individual image depth for point sources. Please differentiate between motivation for a desired sky brightness or individual image depth (as calculated for point sources). Please provide sky brightness or image depth constraints per filter.

Ideally twilight images would have exposure time(s) such that they achieve the same depth as a single-visit WFD exposure. 


\subsection{Co-added image depth and/or total number of visits}

Constraints on the total co-added depth and/or total number of visits. Please differentiate between motivations for a given co-added depth and total number of visits. Please provide desired co-added depth and/or total number of visits per filter, if relevant.

There is no constraint on co-added depth. The suggestion is for a minimum of 1-2 visits in each sky region over the lifetime of the survey. Determining the optimal number (and airmass) of these visits requires further modeling.

\subsection{Number of visits within a night}

Constraints on the number of exposures (or visits) in a night, especially if considering sequences of visits.

A minimum of one visit per night in which the telescope takes any observations.

\subsection{Distribution of visits over time}

Constraints on the timing of visits - within a night, between nights, between seasons or between years (which could be relevant for rolling cadence choices in the WideFastDeep. Please describe optimum visit timing as well as acceptable limits on visit timing, and options in case of missed visits (due to weather, etc.). If this timing should include particular sequences of filters, please describe.

If adopting a twilight survey, each WFD pointing should be observed a minimum of 1-2 (additional) times during the course of the 10-year survey.

\subsection{Filter choice}

Please describe any filter constraints not included above.

DCR will only be detectable in the $u$ - and $g$-bands, thus it is crucial to maximize the number of epochs of observation in those filters. In the case of twilight observations, the baseline suggestion is to limit to $g$-band only. However, additional $u$-band and $r$-band observations would improve the DCR model in each tract in those bands; this would result in improved difference imaging due to fewer false positives and would provide better sub-band fluxes for template fitting (both quasar and stellar).

\subsection{Exposure constraints}

Describe any constraints on the minimum or maximum exposure time per visit required (or alternatively, saturation limits). Please comment on any constraints on the number of exposures in a visit.

Exposure time needed to achieve nominal WFD depth during twilight.

\subsection{Other constraints}

Any other constraints.

The minimum airmass for additional observations must be $\geq 1.5$. 


\subsection{Estimated time requirement}

Approximate total time requested for these observations, using the guidelines available at https: //github. com/lsst-pst/survey_strategy_wp.

We assume exposure times of no more than 60 s and $87 g$-band visits over 10 years (in baseline2018a). If only a single visit needs to be at $\mathrm{AM}>1.5$, then that is just $1 \%$ of the visits with no extra time required. If adopting a twilight survey, and assuming that roughly 2000 pointings are needed to tile the WFD area, we estimate that the amount of twilight time is no more than about 70 hours (for 2 additional visits). Roughly speaking, one in every 10 clear nights would need $<60$ s $g$-band exposure to be taken during the morning or evening twilight.

\begin{tabular}{l|l|}
\hline Properties & Importance \\
\hline Image quality & 2 \\
Sky brightness & 1 \\
Individual image depth & 1 \\
Co-added image depth & 3 \\
Number of exposures in a visit & 3 \\
Number of visits (in a night) & 3 \\
Total number of visits & 1 \\
Time between visits (in a night) & 3 \\
Time between visits (between nights) & 3 \\
Long-term gaps between visits & 3 \\
Other (please add other constraints as needed) & \\
\hline
\end{tabular}

Table 1: Constraint Rankings: Summary of the relative importance of various survey strategy constraints. Please rank the importance of each of these considerations, from $1=$ very important, $2=$ somewhat important, $3=$ not important. If a given constraint depends on other parameters in the table, but these other parameters are not important in themselves, please only mark the final constraint as important. For example, individual image depth depends on image quality, sky brightness, and number of exposures in a visit; if your science depends on the individual image depth but not directly on the other parameters, individual image depth would be ' 1 ' and the other parameters could be marked as ' 3 ', giving us the most flexibility when determining the composition of a visit, for example.

\subsection{Technical trades}

To aid in attempts to combine this proposed survey modification with others, please address the following questions:

1. What is the effect of a trade-off between your requested survey footprint (area) and requested co-added depth or number of visits? 
2. If not requesting a specific timing of visits, what is the effect of a trade-off between the uniformity of observations and the frequency of observations in time? e.g. a 'rolling cadence' increases the frequency of visits during a short time period at the cost of fewer visits the rest of the time, making the overall sampling less uniform.

3. What is the effect of a trade-off on the exposure time and number of visits (e.g. increasing the individual image depth but decreasing the overall number of visits)?

4. What is the effect of a trade-off between uniformity in number of visits and co-added depth? Is there any benefit to real-time exposure time optimization to obtain nearly constant single-visit limiting depth?

5. Are there any other potential trade-offs to consider when attempting to balance this proposal with others which may have similar but slightly different requests?

If extending the WFD observing season so as to achieve some epochs at higher airmass, there could be a trade-off in depth vs. the nominal WFD depth if exposure times are not adjusted accordingly.

If instead a twilight survey is implemented, then at 60s/night for a twilight survey outside of the standard cadence, there really are no trade-off issues, other than competing twilight proposals. 


\section{Performance Evaluation}

We have simulated both the colors of quasars and their DCR offsets as a function of redshiftincluding the appropriate covariances. We are then able to create mock photometric and astrometric data based on opSim cadences (and thus airmasses).

The metrif 1 that we have adopted is the standard deviation within the interquartile range as a function of redshift as shown in the left-hand panel of Figure 3. Improvements in photometric redshifts can be measured/visualized as a reduction in the standard deviation. In the right-hand panel of Figure 3 we also show a histogram of redshift errors (at all redshifts), which makes the photo- $z$ improvement that comes from adding DCR somewhat clearer (although with the loss of information about the accuracy at specific redshifts).
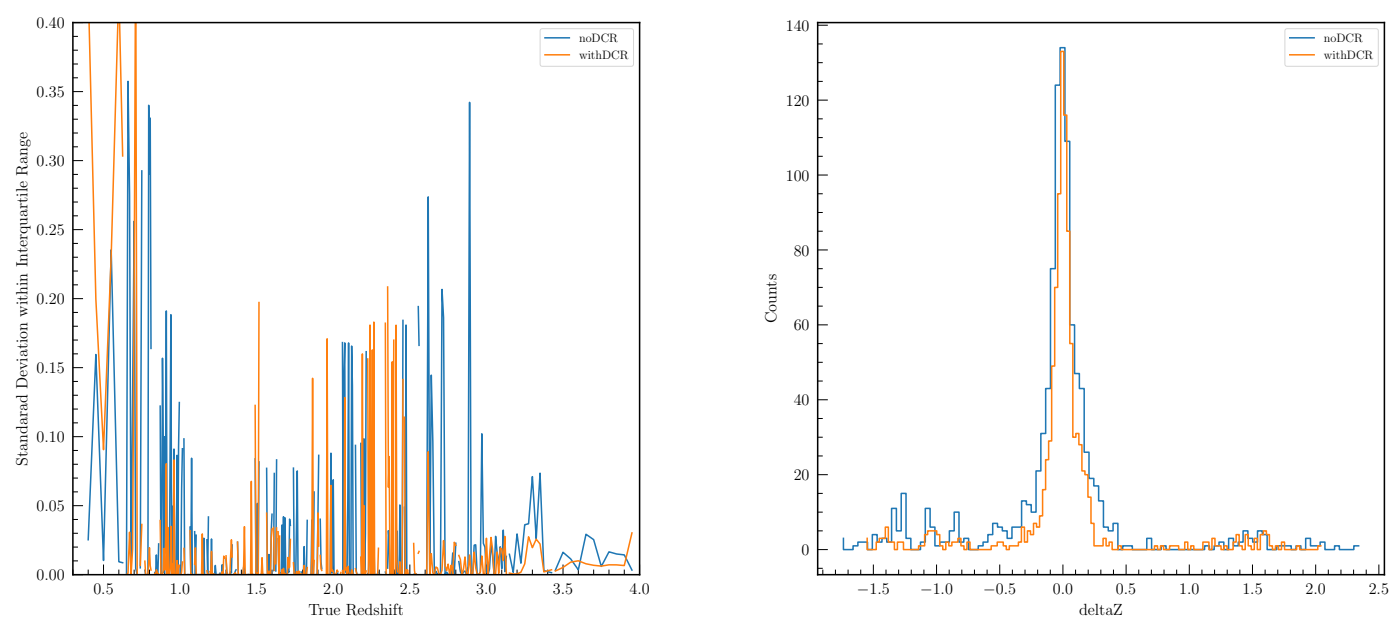

Figure 3: Robust standard deviation of our photo-z estimates as a function of true redshift. Spikes in the plot indicate redshifts at which the prediction varies more widely from the true redshift. A more accurate prediction would produce fewer spikes and more values near or at zero. The metric computed using photo- $z$ derived without including DCR information is shown in blue, including DCR for the nominal WFD is shown in orange. Further simulations are needed to determine what additional observations are needed to optimize the impact of DCR on the improvement of photo- $z$ (and thus also object classification).

Figure 4 illustrates how additional airmass observations are utilized. The slope of the DCR effect with airmass can be thought of as similar to a "color" (which is redshift dependent). More accurate estimation of the slope therefore leads to improvement of photo- $z$

\footnotetext{
${ }^{1}$ The core code for the metric (but not including some large data files) is available at https://github.com/RichardsGroup/LSSTprep/blob/master/DCR/TestAstrometryMetricwithoutMAFV4.ipynb
} 
estimates and having a longer lever arm in airmass (here shown as the tangent of the zenith angle) increases the accuracy of the slope estimate.

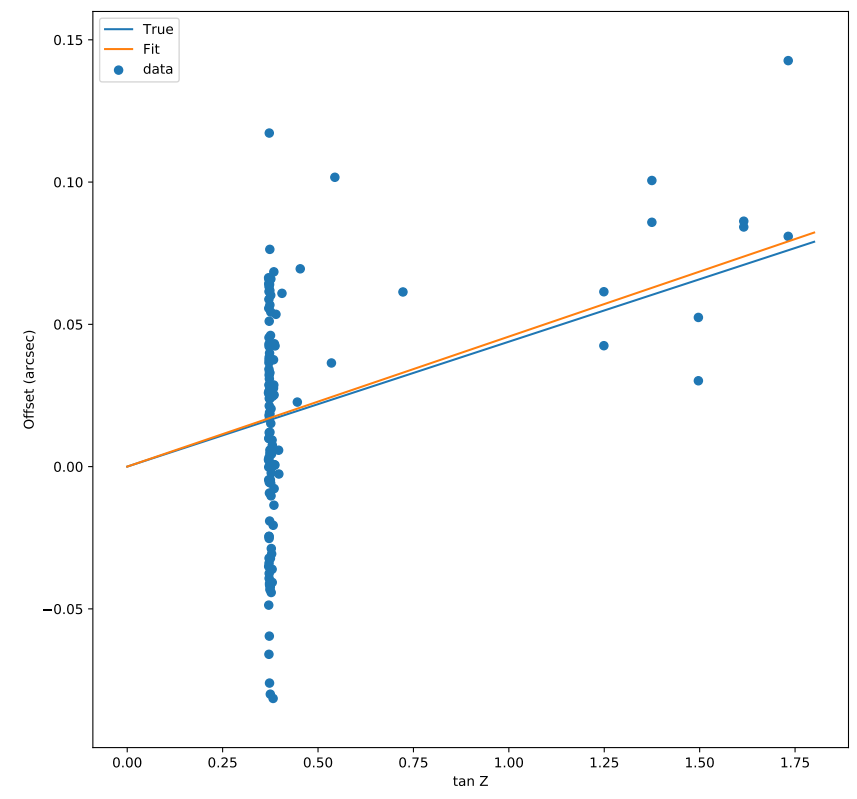

Figure 4: DCR Positional offset ( $g$ band) vs. $\tan Z$ (which is related to airmass, but is linear with the expected DCR offset). The slope of the DCR effect with airmass is shown in blue. The best fit is shown in orange. Here we have improved the fit by adding 2 epochs at each of airmasses 1.6, 1.7, 1.8, 1.9, and 2.0-for illustrative purposes.

When adding DCR information, we first estimate the slope of the positional offset in both the $u$-band and $g$-band as a function of airmass. This slope is compared to the known mean slopes as a function of redshift in the same way that we compare the measured color to the mean color as a function of redshift. Thus both colors and DCR provide measurements that are independent, but that the algorithm treats in a similar manner.

Our analysis is differential, so the method of estimating photo- $z$ is not all that important, but for the sake of completeness, we are using the empirical (not template-based) method of Weinstein et al. (2004). We compute the full photo- $z$ PDF for each object and convert that to a single photo- $z$ point estimate. The point estimate is calculated by determining which of the peaks in PDF have the most area under the curve, then choosing the redshift that corresponds to the highest probability in that peak.

Further modeling is needed to determine the optimal parameters of a twilight survey (or modifications to the baseline cadence), the parameters being number of epochs, airmass, and bandpasses. Our code provides the framework for such an analysis. 


\section{$5 \quad$ Special Data Processing}

Describe any data processing requirements beyond the standard LSST Data Management pipelines and how these will be achieved.

Given the minimal time requirement, if the observations are completed at the beginning of twilight (such that the sky is still fairly dark), the data would effectively be equivalent to all the other WFD observations and could be processed as if they were actually part of the WFD survey. However, the LSST Software Pipeline does not currently model DCR as part of its astrometric fits, so high airmass observations would not have optimal astrometric calibrations. Plans for astrometry include explicitly incorporating DCR effects in the future. The effect of high airmass on coaddition and astrometric repeatability could be explored as part of the DESC simulation data challenges.

\section{References}

Kaczmarczik, M. C., Richards, G. T., Mehta, S. S., \& Schlegel, D. J. 2009, AJ, 138, 19

Lee, M., Budavari, T., Sullivan, I., \& Connolly, A. 2018, ArXiv e-prints, arXiv:1807.07211 [astro-ph.IM]

Peters, C. M., Richards, G. T., Myers, A. D., et al. 2015, ApJ, 811, 95

Sullivan, I. 2018, "DMTN-037: DCR-matched template generation"

Weinstein, M. A., Richards, G. T., Schneider, D. P., et al. 2004, ApJS, 155, 243 